\section{RADIANT LIGHT AND HEAT}

\section{lV. (Continued)}

\section{Radiation and Absorption-Celestial Applications}

T'HIS is perhaps the most suitable place for alluding to a method of obtaining a picture of the corona on ordinary occasions, recently introduced by Dr. Huggins, and which has already met with considerable success.

By using a suitable absorbing medium Huggins has been able so greatly to diminish the proportion between the terrestrial glare and the light from the corona, that a photographic image of the regions around the sun exhibits visible traces of an excess of action in certain places which are probably those occupied by the corona. Plates prepared in this manner were compared with those taken of the corona in Egypt during a total eclipse, and the comparison, made by several observers, appears to leave little doubt that the object photographed is really the corona. A development of this method would prove a great boon to solar inquiry.

Another result of the application of the spectroscope to the sun has been the determination of the rates of motion of the currents which take place in the solar atmosphere.

This is done by the method of displacement already mentioned, a motion of solar gas towards the eye pushing its spectral lines to the more refrangible side of their ordinary position, while a motion in the opposite direction has a contrary effect. In Fig. 20 we have a representation of the deviation of the $F$ line in a spot spectrum.

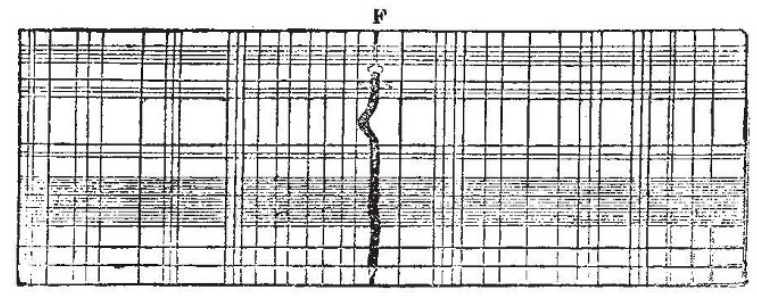

FIG. 20.

By this means prodigious solar velocities have been observed. In our earth when air moves at the rate of Ioo miles an hour we count it a hurricane, but in the sun we find gaseous matter frequently moving at the rate of 100 miles a second.

It has likewise been observed that the velocities of solar motions are greatest on occasions of maximum sun spots, when there appears to be a general increase in the activity of all things belonging to our luminary.

It may therefore be said that all our observations combine in proving how extensive the solar atmosphere must be, and how enormous must be the ve!ocities of its constituents, and more especially of the hydrogen, which enters largely into its composition.

We have thus two facts connected with our luminary which fill us with amazement. For we have, in the first place, his continued ability to radiate powerfully without cessation, or even apparent diminution, and we have, in the second, the astounding velocities of his atmospheric motions.

A little reflection will, however, serve to convince us that these two wonderful facts are intimately connecter together and serve to explain each other, and that in truth the atmospheric motions are the very machinery which enables the sun to continue his radiation.

For let us inquire what is the essential condition of such continued radiation.

Clearly we must have some process by which there shall be a continuous and very rapid stream of fresh

$$
x \text { Continued fron p. } 38 \text {. }
$$

particles sent to the surface of the sun. These are there required to give out their light and heat, and then promptly to retire, being replaced by fresh particles from beneath, which again in their turn give out light and heat and then rapidly retire. It is necessary that there should be some powerful machinery of this kind, in virtue of which fresh recruits shall continually be carried to the front, while the exhausted battalions are promptly marched behind into the magazine.

Now such machinery is supplied in the vast and intense solar convection currents by means of which the cold matter from above is rapidly carried down, forming a sun spot, while the hot matter from beneath is rapidly carried upwards, forming a faculı.

This ceaseless system of ascending and descending currents gives rise, no doubt, to the mottled appearance of our luminary, while in certain districts of the sun and on certain occasions the system is swelled out into gigantic proportions, and we have a large sun spot, with its accompanying faculæ. Nor is it difficult to understand why convection currents should be so powerful in the atmosphere of our luminary. The intensity of such currents will depend upon the following conditions :- -

(I) Cn the intensity of the heat of the hot portions of the arrangement as compared to that of the cold.

(2) On the intensity of gravity.

(3) Un the scale of the whole arrangement.

(4) Cn the presence of condensible substances in the atmosphere.

Now in the sun the heat of the hot particles is very great, while the space around the sun may be taken to represent something without heat. Again the intensity of gravity at the sun's surface is very great, being about twenty-eight times greater than that with which we are familiar on the earth.

In the third place, the scale of the whole arrangement is very great; and, lastly, we have without doubt the presence of condensible substances in the solar atmosphere. All these are powerful causes, and we must bear in mind that they have not merely to be added, but rather multiplied together. Can we therefore wonder that their joint effect is such as to raise the violence of solar storms into something like 60 or 100 miles per second?

These considerations may likewise, perhaps, serve to throw light on the question of solar variability. We have seen that sun spots have a period of eleven years, and that near the minimum of this period there are occasions when the sun is entirely without spots.

Now it is sufficiently obvious, and has likewise been proved experimentally, that a sun spot gives out less light and heat than the ordinary solar surface. On the other hand, the proportion which the spotted area bears to that of the whole disc is insignificant, so that, taking these two facts together, we should at first sight imagine that the sun ought to give us very slightly less light and heat on those ocrasions when there are most spots.

I think, however, that this direct action is probably inappreciable, and that sun spots are rather to be regarded as symptoms of a particular state of the sun, implying an increased activity of solar convection currents. Now, inasmuch as the outpouring of solar light and heat is kept up by means of these convection currents, we might therefore expect that on occasions when such currents are peculiarly powerful the sun should give out most light and heat. "To use the words of the late Sir J. Herschel, the "sun pot" may on such occasions be boiling very rapidly.

On the whole, therefore, theory would lead us to infer that the sun will be found most powerful in its radiation on those occasions when it has most spots on its surface. It will, however, be noticed that this is merely a theoretical conclusion, and has to be supported by evidence which must of course be terrestrial. Have we, then, any terrestrial evidence that the sun is more powerful in its 
radiation at times of maximum than at times of minimum sun spot frequency?

In reply to this question, we must acknowledge that of direct evidence derived from the actinometer we have hardly any. Certain preliminary observations made by Mr. Hennessey at the headquarters of the Trigonometrical Survey in India may perhaps induce us to imagine that the sun may be most powerful on occasions of maximum sun spot frequency, but this is far from conclusive. We have, however, very strong indirect evidence in favour of this conclusion. This is derived partly from the facts of terrestrial magnetism and partly from those of terrestrial meteorology, that from the former being the stronger of the two. It is well known that the sun produces changes in the magnetism of the earth. It gives rise, for instance, to the solar-diurnal variation of the needle which is a systematic change ; and it likewise produces magnetic storms, these words being employed to denote changes of a peculiarly abrupt and irregular kind. Now undoubtedly these both imply an energetic action of some kind on the part of the sun, and we have strong grounds for supposing that this energetic action is connected with the radiating power of our luminary. But both of these solar actions upon the magnetism of the earth are decidedly stronger in times of maximum than in times of minimum sun spot frequency.

Such electrical phenomena as the aurora borealis and the currents which take place in the crust of the earth are likewise peculiarly developed on the same occasions.

In Fig. 21 we have a diagram representing the connection between sun spot maxima, the maxima of declination range, and the frequency of the aurora borealis.

When we come to meteorology, the evidence before us is not so conclusive, although here also what we have tends, I think, in the same direction. Mr. Meldrum has shown that there are most cyclones in the Indian Ocean

\section{Solar Spots, Magnetic Declination,and Auroral Displays,}

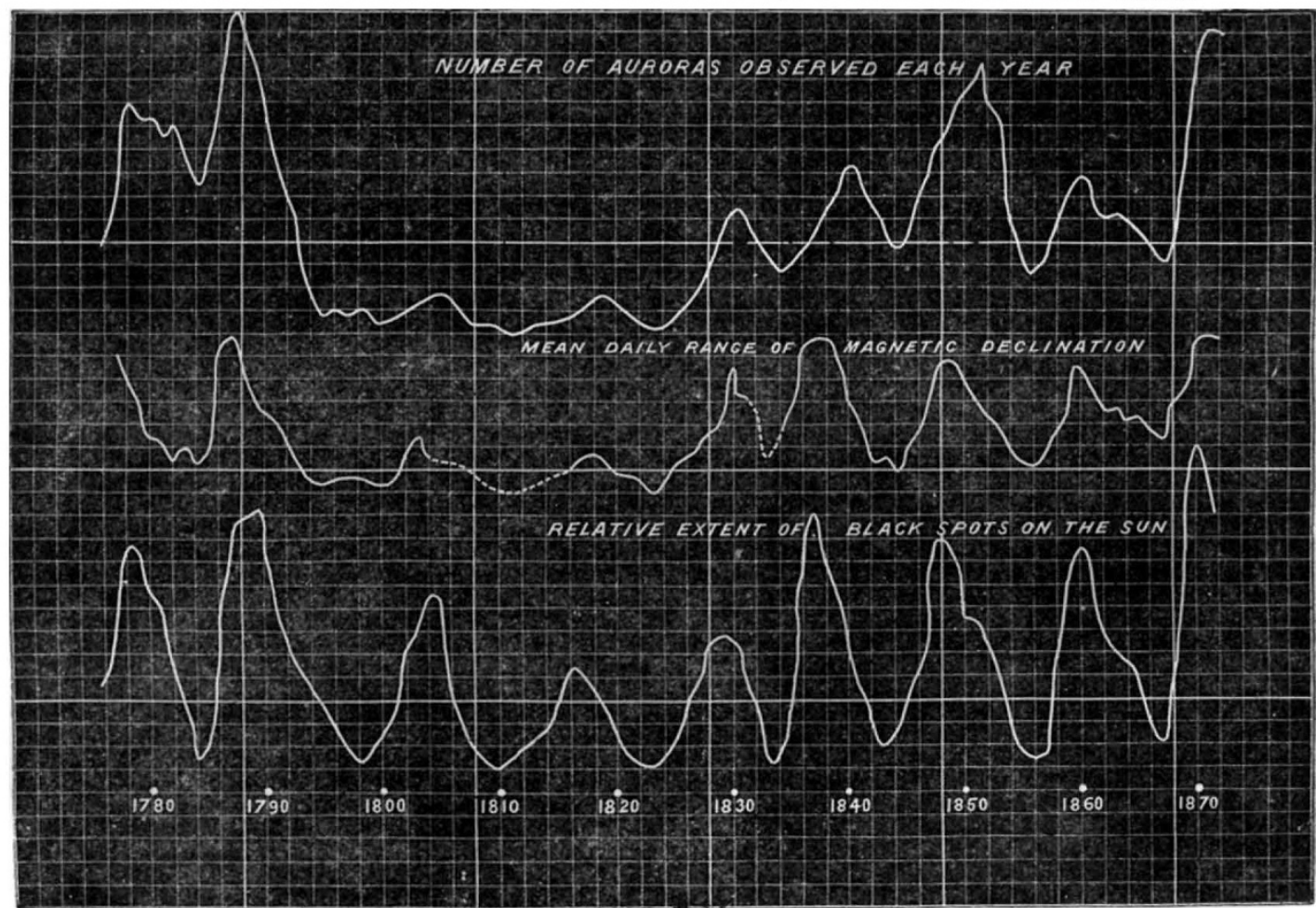

FIG. 2I.

about times of maximum sun spots, and M. Pöey has proved the same thing with respect to West Indian hurricanes. On the whole, too, and in the majority of stations, the rainfall is greatest on the same occasions.

Again, let us take the barometric pressure of the air. Here a little reflection will convince us that the peculiar variations in the distribution of such pressure are really caused by the sun. For instance, we know that Western Siberia during the winter season has a pressure decidedly above the average, and we should therefore imagine that in years when the sun is peculiarly powerful the winter pressure in Siberia would prove to be particularly high. Now this is just the state of things which Mr. Blanford has found to correspond with years of maximum sun spot frequency, and thus the evidence is in favour of these being also years of maximum solar power. The Indian meteorologists have derived similar conclusions from the observations made in India. In fine, we may, with the balance of probability in our favour, adopt the conclusion deduced by Mr. Baxendell at a comparatively early period, who found that the forces which produce the movements of the atmosphere were apparently more energetic in years near maximum than in years near minimum sun spot frequency.

Let me now proceed to indicate the nature of the information which the spectroscope gives us regarding the planets and comets of our system. Since the moon and the various planets are illuminated by the sun, their spectra will necessarily be built upon that of the sun. As a matter of fact, this is found to be the case ; but we ought to bear in mind that the solar rays that reach us from a planet must have penetrated some distance into the atmosphere of that planet. In doing so they will most probably have suffered spectral absorption, the 
nature of which may thus suffice to throw light upon the constitution of the planet's atmosphere.

Working in this manner, Dr. Huggins has observed no trace of an atmosphere in the moon, but in the spectrum of Jupiter lines are seen which indicate the existence of an absorbing atmosphere. One band appears to correspond in spectral position with dark lines due to our earth's atmosphere; but another band is different from

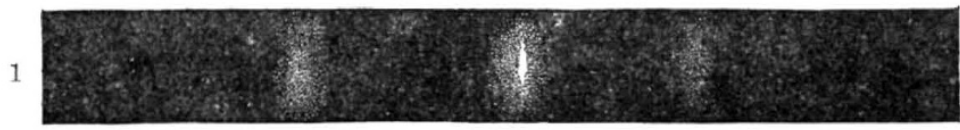

2

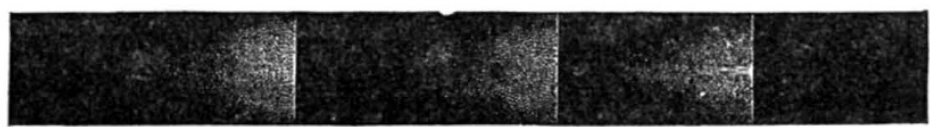

3
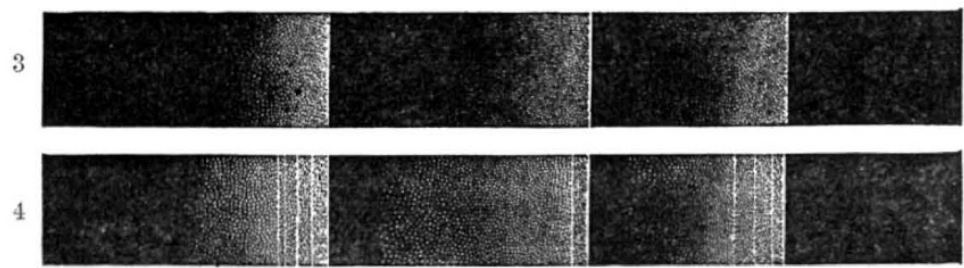

Fig.n22.-(1) Spectrum of Brorsen's comet ; (2) Spectrum of Winnecke's comet; (3) Spectrum of carbon in olefiant gas; ( 1 ) Spectrum of carbon in olive oil (Huggins).

any line caused by our atmosphere, and indicates most aqueous vapour probably exists in the atmospheres of probably the existence of some unknown constituent. Jupiter and Saturn.

Saturn has likewise a band common to the earth, so that The spectrum of Mars indicates in like manner the

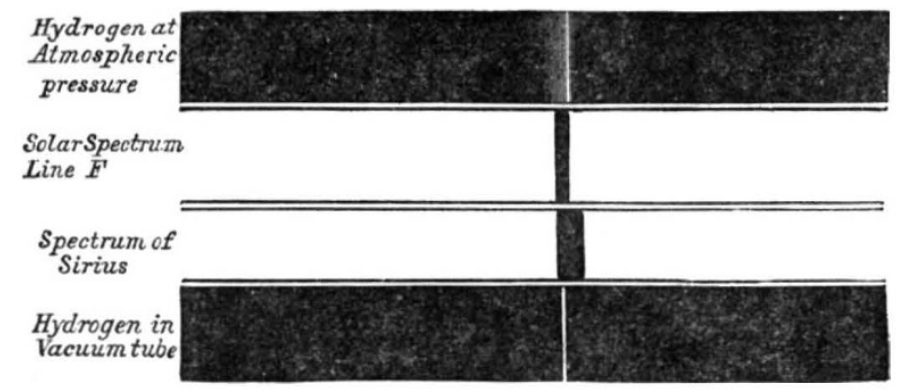

FIG. 23.

existence in the atmosphere of that planet of matter similar to that which occurs in the earth's atmosphere.

The absorption spectra of the far distant planets hav likewise been examined.
Padre Secchi and M. Janssen agree with the conclusion that the vapour of water probably exists in certain planetary atmospheres.

In the absorption spectrum of our atmosphere Professor

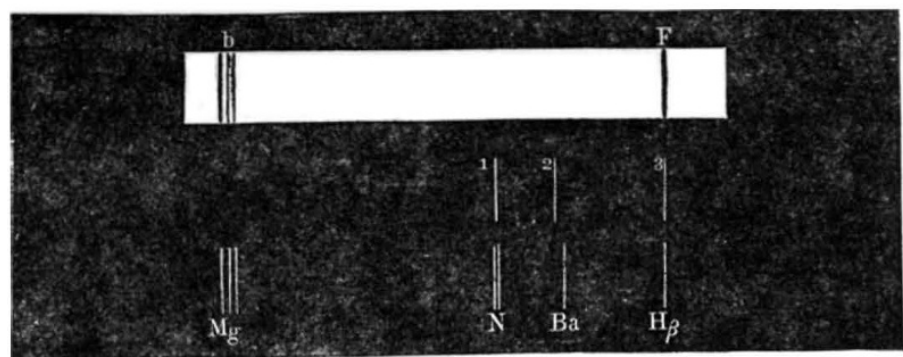

FIG. 24. First, the solar spectrum; next, the spectrum of the nebulæ; $x, 2,3$ the lines observed. Below, the bright lines of magnesium, nitrogen, barium, and hydrogen.

Piazzi-Smyth has noticed a band which appears to be from our atmosphere, there is a line which we cannot associated with the presence or possibility of rain, and which he has termed the rain-band. Strangely enough in the bright spectrum of the aurora borealis, which comes identify with the spectrum of any known terrestrial substance.

Dr. Huggins has likewise studied the spectra given by 
the brighter portions of comets, and has obtained for many of these bodies lines which resemble the spectrum of carbon, as taken in a hydrocarbon.

In Fig. 22 we have, first, the spectrum of Brorsen's comet; secondly, the spectrum of Winnecke's comet; thirdly, the spectrum of carbon in olefiant gas ; fourthly, that of carbon in olive oil.

More recently the same observer has found other specimens of this class of bodies, which give spectra essentially different from the hydrocarbon type, and he remarks that as the meteors which come down to us differ greatly in chemical constitution, so it is ot surprising that a similar difference should be found in comets which we know to be very closely allied to meteors.

Dr. Miller and Mr. Huggins were amongst the first to give us information regarding the spectra of stars. These bodies exhibit spectra very similar to that of the sun-that is to say, they give us an underlying continuous spectrum, intersected with dark lines. We have thus evidence of a similarity in physical constitution between our sun and these very distant bodies.

The position of the dark lines is the great object of interest in stellar spectra, and by the method already described the presence of various terrestrial elements has been detected in the stars.

In the most brilliant bluish-white stars, along with the presence of numerous fine lines, we have a comparatively small number of prominent absorption lines, the substances which these indicate as present being hydrogen, calcium, magnesium, and sodium. We shall return to this subject on a future occasion.

Miller and Huggins were so fortunate as to obtain the spectrum of a star which suddenly blazed out in May, 1866 , and found in it the unmistakeable presence of bright hydrogen lines.

Such a star is probably to be regarded as exhibiting on an enormous scale the same phenomenon which is frequently seen in the outburst of hydrogen from the interior of our luminary. Indeed, we have sometimes bright lines of hydrogen in the spectrum of certain solar regions. Padre Secchi has also ascertained that several very small stars exhibit bright lines.

I need hardly say that in the case of the fixed stars where the disc is a mere point, we have no possibility of differentiating between various portions of it, or of ascertaining the velocities of its atmospheric motions by spectral displacement.

We have, however, the means of ascertaining by this method whether the star be approaching us or receding from us, and how rapidly it is doing so. Huggins has made many laborious and interesting observations of this nature, and has determined the relative motion to or from us of many stars-a result which could not possibly be ascertained without the spectroscope.

In Fig. 23 we have an indication of the proper motion of Sirius as seen by the displacement of the hydrogen line $\mathrm{F}$ in the spectrum of that star.

Before concluding this branch of my subject, let me briefly allude to the light thrown by spectrum analysis on the composition of certain of the nebulæ. On directing his stellar spectroscope to the planetary nebula in the constellation Draco on 2oth August, 1864, Dr. Huggins found that its spectrum consisted of three bright lines on a dark background-in fine, that it was the spectrum of incandescent gas.

Of these three lines one is in all probability the hydrogen line $\mathrm{H}$; another appears to coincide with one of the lines of nitrogen, while the third does not coincide with any known line.

Other nebulæ have since been found by Huggins to give us similar spectra.

Fig. 24 denotes the general spectrum given by the nebulæ.

It thus appears that we have already derived great information regarding the constitution of the heavenly bodies by means of spectrum analysis.

BALFOUR STEWART

(To be continued.)

\section{NOTES}

THE death is announced, in his seventy-first year, of Dr. John Christopher Draper, Professor of Chemistry in the Medica Department of the University of the City of New York. Dr. Draper was the eldest son of the eminent Prof. John W. Draper. His scientific papers, apart from those on the science of medicine, are devoted to chemical and physical subjects ; and among the latter chiefly to optical phenomena. His last one, relating to dark lines in the solar spectrum, attracted some attention at the time, $1878-79$.

THE death is announced of Mr. Jamẹs Fergusson, F.R.S., on the $9^{\text {th }}$ inst., at the age of seventy-eight years. Mr. Fergusson was well known by his writings on Indian architecture, and also by his magnificent work on "Tree and Serpent Worship in India."

THE deaths among French Academicians have been unusually numerous of late; we have to record this week that of $\mathrm{M}$. de Saint-Venant, a member of the Section of Geometry.

THE Russian Academy of Science has elected Mr. David Gill, Astronomer-Royal at the Cape of Good Hope, a Corresponding Member.

SEVERAL French papers have published articles on the opportunity of celebrating the centenary of Arago's birth, this celebrated astronomer and physicist having been born at Estagel, a country town of the Department of the Pyrénées Orientale-, on Feb. 26, 1786. As he was a Copley Medallist and a foreign member of the Royal Society of London, it is expected that this Society will be invited to send a delegate to take part in the proceedings. Arago was elected a member of the Paris Academy at the early age of twenty-three, after having achieved the measurement of the meridian arc from Dunkirk to Formentera, for determining the length of the metre. His predecessor was Jerome de Lalande. In 1830 he was elected Perpetual Secretary, and he continued to act as such during twenty-three years up to his death, which took place in 1853 . His works have been edited by Barral, and fill seventeen large octavo volumes, of which four are devoted to "Astronomie Populaire."

IN a very excellent article in Science on "The Government and its Scientific Bureaus," we find some wholesome remarks un the conditions under which scientific work can be performed at its best. "Science cannot be carried forward by prescribing too definitely the tasks of scientific men. They may be bound by appointed days and hours; they may be told to perform specilic duties, - and if only the maintenance of routine work is required, such regulations may secure fidelity and efficiency. But if knulvledge is to be advanced, if better methods of work are to be discovered, if greater accuracy is desired, if unknown facts are to be ascertained and recorded and discussed, and, in short, if there is to be real progress, the methods of freedom are to be employed, not those of petty regulation. By this we mean that if the great undertakings which the Government has in charge, if especially its surveys of the coast and of the interior are to gu forward, discretion must be given to the chiefs of bureaus, and they must be held to accountability for the aggregate succes, of their work. Honesty, economy, clear and accurate statement of accounts are, of course, to be demanded in every office : nobody questions this. But the determination of what shall be uudertaken in a given year, to whom it shall be assigned, what allowances shall be made for instruments, books, and assistants, - these are questions which experience and judgment must decide. Somebody who has all the facts in mind must make 